\title{
Systematic Investigations of Plastic Vials Concerning Their Suitability for Ultratrace Anion Analysis in High-Purity Industrial Applications
}

\author{
Renato Figi*, Claudia Schreiner, and Davide Bleiner \\ Swiss Federal Laboratories for Materials Research (EMPA), Ueberlandstrasse 129, CH-8600 Dübendorf, Switzerland
}

Received September 19, 2004; accepted March 15, 2005; published online June 9, 2005

(C) Springer-Verlag 2005

\begin{abstract}
Ultratrace anion analysis in high-purity industrial applications is done using commercially available plastic vials to store the eluted liquid samples. Plastic vials are manufactured with materials containing several additives. Such additives might potentially release anionic contaminants, thus degrading the blank quality and determination limits (DL). The durability and thermal stability of several materials was found to be a function of anionic species. Fluorinated materials showed the best results for the majority of anions with the exception of fluoride. For the selective analysis of fluoride, polypropylene was found to be the best vial material, together with glass. Cold water extraction showed better results compared to hot water extraction. Over a long observation period, hot water extraction did not at all improve the performance, indicating that this type of supposedly efficient cleaning of the plastic containers to render them less prone to contaminant release does not hold true.
\end{abstract}

Key words: Ion chromatography; sample vials; high purity water analysis; SEMI F40/F57; ultratrace anions.

In the semiconductor industry, pipeline water must be periodically analyzed for quality control. In fact, the plastic components of the piping systems are likely to

\footnotetext{
* Author for correspondence. E-mail: renato.figi@empa.ch
}

release anions such as chloride $\left(\mathrm{Cl}^{-}\right)$, phosphate $\left(\mathrm{PO}_{4}{ }^{3-}\right)$, and sulphate $\left(\mathrm{SO}_{4}{ }^{2-}\right)$ which even at ultratrace level are corrosion-promoters for electronic components. Semiconductor Equipment and Materials International (SEMI) has regulated the tolerance of such contaminants, indicating extraction procedures and threshold limits for all trace components, i.e. anions, cations and total organic carbon (TOC). Quality assessment of high-purity components is performed in elution tests, determining the amount of released anions, cations, and TOC. This procedure is regulated in standard operating procedures (SOP), such as the American Society for Testing and Materials (ASTM) [1], or the well-accepted SEMI F40 and SEMI F57 [2, 3].

The annual book of the ASTM standards [1] suggests the following procedure: filling the vials completely with high-purity water, capping, and allowing to soak for 1 day up to 5 days maximum. After filling, placing the vials in polyethylene (PE) bags and keeping them sealed during the storage period. The highpurity water should be changed at a five-day-interval during long storage periods. Recommended materials are high density polyethylene (HDPE), low density polyethylene (LDPE), polysulfone (PSU), polycarbonate $(\mathrm{PC})$, polystyrene (PS), polypropylene (PP), and polymethyl-pentene (PMP, TPX ${ }^{(\mathbb{B})}$ ). Unfortunately, this 
procedure does not provide information about the eluted concentration of the anions of interest as function of time. Moreover, the suggested materials are characterized by different resistance to pure water aggression.

According to SEMI SOP's F40 and F56 [2, 3], samples are eluted in a drying oven at $85^{\circ} \mathrm{C} \pm 5^{\circ} \mathrm{C}$ in perfluoralkoxy-copolymer (PFA) vessels of $200 \mathrm{~mL}$ or $500 \mathrm{~mL}$ (Savillex ${ }^{\circledR}$, USA) for 7 days using highpurity water (specific resistivity $>18 \mathrm{M} \Omega \mathrm{cm}^{-1}$ at $25^{\circ} \mathrm{C}$ ). The eluted aqueous solutions are filled into sample vials to perform analytical determinations by means of ion chromatography (IC). The sample volume available for anion determination is limited to just $10 \mathrm{~mL}$ because according to the SOP other portions of the eluted sample must be used for concomitant determination of cations by means of ICPMS, and total organic carbon (TOC) by means of combustion methods. The sample volume for the measurement is also limited because a large part of the total sample volume is used for rinsing the measurement vials. Reduced sample volume introduces concerns on sample representativity and determination limits (DL), due to less dilution of potential contamination from the vials.

Measurements performed with large sample volumes, even at low anion concentration level, are less influenced by potential contamination deriving from sample containers. Nguyen [4] described a procedure for online anion enrichment (up to $130 \mathrm{~mL}$ enrichment volume) which also guarantees eluted sample purity since no blank or sample storage is necessary. This results in low DL, approx. in the $n g L^{-1}$ range.

In fact, the purity of the blank is often the most limiting factor in the effort to obtain lower and lower DL. Nguyen et al. [5] also reported on a storage procedure in pre-cleaned FEP containers at $7{ }^{\circ} \mathrm{C}$ in darkness, yet the details about the pre-cleaning procedure and its efficacy were not fully discussed. Only a selection of the list of anions recommended in the SEMI F40 procedure is given, but information about the highly FEP-concentrated fluoride is missing.

Thus, concerns about anion contamination introduced by plastic sample containers or vials are of primary importance in high-purity industrial applications where strictly as low as $10 \mathrm{~mL}$ are available for IC determinations. Commercially available laboratory equipment, e.g. sample vials for auto-sampler systems, should be contaminant-free, in order to allow reliable analytical determinations. If, on the contrary, these yielded anions as a function of time, the consequence would be a degradation of analytical results, blank values, and DL. Furthermore, directives from the industrial semiconductor-competence centres for lower and lower DL suggest conducting detailed investigation on the suitability of materials used for sample handling and storage, yet this has so far not been done systematically.

A few papers focus on one single material [6] but unfortunately a thorough investigation of material performance has not been presented. Besides, it should be said that commercially available sample vials for conventional auto-sampler systems are mass produced, which is a practice typically not compliant with high-purity concerns as required in ultratrace analysis. In fact, many laboratory equipment manufacturers use plasticizer as an additive which contains anions such as F-, $\mathrm{Cl}^{-}, \mathrm{NO}_{2}{ }^{-}, \mathrm{NO}_{3}{ }^{-}, \mathrm{SO}_{4}{ }^{2-}$. These are potential contaminants.

The aim of this study was to find out what materials for sample handling and storage are most suited for several anionic species. The investigations were done monitoring the time-dependent release of contaminants from the vials, and comparing these observations with the requirements imposed by the semiconductor industry. The volume of the vials is strictly defined by the $10 \mathrm{~mL}$ sample that is available for the determinations. The materials chosen are those used most commonly by manufacturers in the production of commercially available plastic laboratory equipment. Based on the present investigations we defined a rank list concerning material suitability as sample containers for ultratrace anion analysis. For comparison also vials made of AR-glass were investigated.

\section{Experimental}

\section{Samples}

Sample vials of several plastic materials were used for elution tests. The materials selected were among the most widely distributed and commercially available ones. They are listed in Table 1 . The amount of the investigated sample is $10 \mathrm{~mL}$, due to procedure constraints as explained above.

\section{Instrumentation}

All preparation and dilution procedures of the reactants, solutions and additives were done under clean room conditions in a laminar flow-box of class 5 [7], type SKANAIR HFX 180T. A high-purity water system (Millipore UV 10A, USA) was used with an ion 
exchange cartridge (Q-Gard 2) and a rinse cartridge Quantum Ultra-

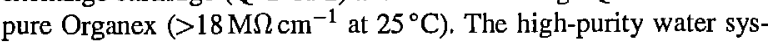
tem was installed in the laminar flow box. All operators were provided with appropriate apparel for the prevention of contamination. The ion chromatography apparatus was installed inside the larninar flow box. The ion chromatography system is composed of a chemically suppressed modular system ( $\Omega$ Metrohm, Switzerland) with the following components: IC pump 709, VA detector 791, IC detector 732, IC separation centre 733, pump unit 752, IC interface 762, IC sample processor 766. Sample pre-concentration was done using a specific cartridge (Metrosep Anion). A Metrosep A 4/5 Guard was used as pre-column, and a Metrohm A-SUPP 4 $250 \mathrm{~mm}$ was used as separation column. The system had been

Table 1. Description of sample vials

\begin{tabular}{|c|c|c|}
\hline Material & Manufacturer & $\begin{array}{l}\text { Vial } \\
\text { dimension } \\
(\mathrm{mm})\end{array}$ \\
\hline $\begin{array}{l}\text { Fluorinated ethylene } \\
\text { propylene (FEP), } \\
\text { Art. ID } 3114-0010\end{array}$ & Nalgene/USA & $\begin{array}{l}\text { H: } 81.5 \\
\emptyset: 16\end{array}$ \\
\hline $\begin{array}{l}\text { Perfluoralkoxy- } \\
\text { Copolymer (PFA), } \\
\text { Art. ID \#8014R }\end{array}$ & Savillex/USA & $\begin{array}{l}\text { H: } 100 \\
\emptyset: 16\end{array}$ \\
\hline $\begin{array}{l}\text { Polytetrafluorethylene } \\
\text { (PTFE), } \\
\text { Art. ID N/A* }\end{array}$ & Semadeni/Switzerland & $\begin{array}{l}\text { H: } 110 \\
\varnothing: 16\end{array}$ \\
\hline $\begin{array}{l}\text { Polypropylene (PP), } \\
\text { Art. ID } 6.2743 .050\end{array}$ & Metrohm/Switzerland & $\begin{array}{l}\text { H: } 110 \\
\emptyset: 16\end{array}$ \\
\hline $\begin{array}{l}\text { Polymethylpentene } \\
\text { (TPX), } \\
\text { Art. ID N/A }\end{array}$ & Semadeni (Switzerland) & $\begin{array}{l}\text { H: } 107 \\
\varnothing: 16\end{array}$ \\
\hline $\begin{array}{l}\text { Polystyrene (PS), } \\
\text { Art. ID N/A }\end{array}$ & Semadeni (Switzerland) & $\begin{array}{l}\text { H: } 95 \\
\varnothing: 16\end{array}$ \\
\hline $\begin{array}{l}\text { Copolymer PP/PE } \\
\text { (PPCO), } \\
\text { Art. ID } 3110-0120\end{array}$ & Nalgene/USA & $\begin{array}{l}H: 100 \\
\varnothing: 16\end{array}$ \\
\hline $\begin{array}{l}\text { Polycarbonate (PC), } \\
\text { Art. ID } 3117-0120\end{array}$ & Nalgene/USA & $\begin{array}{l}H: 101 \\
\emptyset: 16\end{array}$ \\
\hline $\begin{array}{l}\text { Glass } 950 / 1 \\
\text { (AR-Glass), } \\
\text { Art. ID N/A }\end{array}$ & $\begin{array}{l}\text { Assistant/VWR } \\
\text { Switzerland }\end{array}$ & $\begin{array}{l}H: 98 \\
\emptyset: 17\end{array}$ \\
\hline
\end{tabular}

Table 2. Operating conditions for ion chromatography

\begin{tabular}{|c|c|}
\hline Parameter & Specification \\
\hline Separation column & $\begin{array}{l}\text { Metrosep A SUPP } 4, \\
4 \mathrm{~mm} \times 250 \mathrm{~mm}, \\
\text { particle size } 9 \mu \mathrm{m}\end{array}$ \\
\hline Pre-column & Metrosep A 4/5 Guard \\
\hline $\begin{array}{l}\text { Pre-concentration } \\
\text { column }\end{array}$ & $\begin{array}{l}\text { Metrosep anion glass } \\
\text { cartridge } 3.0 \times 30 \mathrm{~mm}\end{array}$ \\
\hline Suppression & chemical suppression \\
\hline Eluent & $\begin{array}{l}1 \mathrm{mMol} \mathrm{L}^{-1} \mathrm{Na}_{2} \mathrm{CO}_{3} \\
4 \mathrm{mMol} \mathrm{L}^{-1} \mathrm{NaHCO}_{3} \\
\text { degassed } 5 \mathrm{~min} \text { in Vacuum }\end{array}$ \\
\hline Regenerant & $50 \mathrm{mMol} \mathrm{L}^{-1} \mathrm{H}_{2} \mathrm{SO}_{4}$ \\
\hline Eluent flow rate & $1 \mathrm{~mL} \min ^{-1}$ \\
\hline Enrichment volume & $10 \mathrm{~mL}$ \\
\hline Background conductance & $17.9 \mu \mathrm{S} \mathrm{cm}^{-1}$ \\
\hline
\end{tabular}

developed in-house to be equipped with two serial detection systems, i.e. a conductance detector IC 732 for the anions $\mathrm{F}^{-}, \mathrm{Cl}^{-}$, $\mathrm{NO}_{3}{ }^{-}, \mathrm{Br}^{-}, \mathrm{PO}_{4}{ }^{3-}$ and $\mathrm{SO}_{4}{ }^{2-}$, and an electrochemical detector VA 791 for $\mathrm{NO}_{2}{ }^{-}$for the ultratrace range. The operating conditions are summarized in Table 2.

\section{Methods}

The eluent was characterized by the following composition: $1 \mathrm{mMol} \mathrm{L}^{-1} \quad \mathrm{Na}_{2} \mathrm{CO}_{3}$ Suprapur and $4 \mathrm{mMolL}^{-1} \mathrm{NaHCO}_{3}$ p.a. (Merck, Germany). The regeneration solution required for the suppressor is composed of $50 \mathrm{mMolL}^{-1} \mathrm{H}_{2} \mathrm{SO}_{4}$ ultrapure (Merck, Germany). Certified Merck ion solutions with a mass concentration of $1 \mathrm{~g} \mathrm{~L}^{-1}$ were used as standard reference materials for the determination of calibration curves. Figure 1 shows the block diagram of the analytical procedure.

Vial elution was performed according to two methods, assuming different elution speeds:

- Cold water extraction: 10 vials were rinsed five times using cold high-purity water, filled, and sealed with caps. At room temperature $\left(20 \pm 2^{\circ} \mathrm{C}\right)$ they were eluted for a period of $1,7,15,28$ and 78 days, then measured and filled again. The same vials were used throughout the entire elution interval.

- Hot water extraction (SEMI F40): 10 vials were rinsed 5 times with cold high-purity water, filled, and sealed with caps. Then

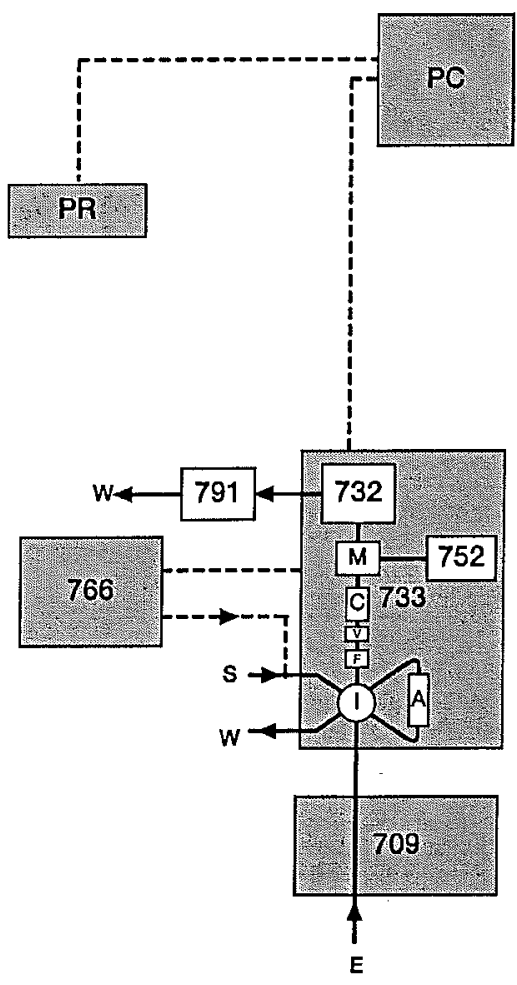

Fig. 1. Block diagram of the ion chromatography system. Legend: $A$ Enrichment cartridge; $C$ separation column (Metrosep A-Supp 4 $250 \mathrm{~mm}$ ); $E$ eluent; $F$ Metrosep RP guard; $I$ injector; $M$ suppressor module; $P C$ computer; $P R$ printer; $S$ sample; $V$ Metrosep A $4 / 5$ guard, pre-column; $W$ waste; 709 IC pump; 732 conductance detector; 733 IC separation center; 752 IC pump unit (suppressor); 766 autosampler; 791 VA detector 
they were stored in a dry oven at $85 \pm 5^{\circ} \mathrm{C}$ and eluted for a period of $1,3,7$, and 15 days, cooled down to room temperature and measured, then filled again.

\section{Results and Discussion}

In the first instance, blank contamination was investigated, and the obtained values were compared to norm threshold values of the SEMI F40. The results for the different anions are shown in Table 3 , indicating that the experimentally observed values match the SEMI F40 threshold values when following the procedure developed. Furthermore, these results confirmed that no contamination was present in the water used for the elution procedure.

Figure 2 shows example chromatograms acquired with the cold extraction procedure for FEP, selected to show IC performance. Cold water extraction experiments demonstrated that none of the materials is inert to anion release during exposure to pure water, so that blank contamination was always observed. Differences were found to the extent of contamination yielded. All fluorine-bearing materials, i.e. FEP, PFA, and PTFE, yielded up to $100 \mu \mathrm{g} \mathrm{L}^{-1}$ of $\mathrm{F}^{-}$as a function of time steadily soaring. Figure 3 shows that FEP and PFA provided increased levels of contamination for the entire 78 days of investigation. After an elution time of 7 days the concentration of fluoride released from PTFE sinks dramatically, whereas only glass introduces less contamination, approx. by $2-3$ orders of magnitude. In the case of PTFE a rapid decrease was observed even in the first week of elution experiments.

The nitrogen-based anions, i.e. $\mathrm{NO}_{2}{ }^{-}$and $\mathrm{NO}_{3}{ }^{-}$, were characterized by a sudden increase after 28 days

Table 3. Comparison of SEMI F40 recommended figures and experimentally observed values

\begin{tabular}{llll}
\hline Anion & $\begin{array}{l}\text { SEMI F40 } \\
\text { threshold }\end{array}$ & $\begin{array}{l}\text { DL blank 3s sample } \\
\text { container PFA (SEMI } \\
\text { F40 elution procedure) }\end{array}$ & $\begin{array}{l}\text { DL high purity } \\
\text { water direct from } \\
\text { the water line }\end{array}$ \\
\hline
\end{tabular}

Mass concentration in $\mu \mathrm{g} \mathrm{L}^{-1}$

\begin{tabular}{lrlc}
$\mathrm{F}^{-}$ & 3,000 & $0.2^{*} \pm 0.2$ & $0.01^{*} \pm 0.001$ \\
$\mathrm{Cl}^{-}$ & 140 & $0.5^{*} \pm 0.4$ & $0.02^{*} \pm 0.002$ \\
$\mathrm{NO}_{2}{ }^{-}$ & 4 & $0.1^{*} \pm 0.05$ & $0.01^{*} \pm 0.001$ \\
$\mathrm{Br}^{-}$ & 4 & $0.1^{*} \pm 0.1$ & $0.01^{*} \pm 0.001$ \\
$\mathrm{NO}_{3}{ }^{-}$ & 4 & $1.3^{*} \pm 0.9$ & $0.1^{*} \pm 0.05$ \\
$\mathrm{PO}_{4}{ }^{3-}$ & 10 & $10^{*} \pm 5$ & $2^{*} \pm 1$ \\
$\mathrm{SO}_{4}{ }^{2-}$ & 10 & $6^{*} \pm 3$ & $1^{*} \pm 0.5$ \\
\hline
\end{tabular}

* Enrichment volume $10 \mathrm{~mL}$.
(Figs. 4, 5). The stability in cold water over longer periods of time is questionable. High-purity water with very low ion mass content (Table 3 ) might in some way modify the surface of the sample vials so that the plastic material becomes receptive and could release contaminants over a long period of time. Thus, plastic material swelling and intra-deformation might be responsible for continuous release of anions into the solution.

Apart from FEP, all vial materials contributed a nitrite concentration up to one order of magnitude higher in the eluted vials over the entire observation time. A qualitatively similar behaviour was found for nitrate, although contamination was more modest by a factor of at least 2 for all plastic vials.

Chloride was found at less significant concentration levels, typically below $10 \mu \mathrm{gL}^{-1}$, apart from PS which introduced up to $27 \mu \mathrm{g} \mathrm{L}^{-1} \mathrm{Cl}^{-}$(Fig. 6). The values of $\mathrm{SO}_{4}{ }^{2-}$ were in a similar range, with minimal time-dependence apart from PS (Fig. 7).

After all these measurements, FEP could be considered the least prone to contamination of all the materials considered for sample vial manufacturing, except with respect to fluoride. For the selective determination of $\mathrm{F}^{-}$, the materials of choice were found to be PP, PPCO, as well as glass. In addition, sample vial preparation consisting of five rinse cycles using cold water is recommended prior to use. Eluate filling in the vials should follow double rinsing. Material performance under cold water extraction could be ranked as follows:

1. FEP, all anions with the exception of $\mathrm{F}^{-}$

2. Glass, all anions with the exception of $\mathrm{NO}_{2}{ }^{-}$, $\mathrm{NO}_{3}{ }^{-}$

3. PTFE, all anions with the exception of $\mathrm{F}^{-}, \mathrm{NO}_{2}{ }^{-}$, $\mathrm{NO}_{3}^{-}$

4. PFA, all anions with the exception of $\mathrm{F}^{-}, \mathrm{NO}_{2}{ }^{-}$, $\mathrm{NO}_{3}{ }^{-}$

5. PP, excellent for $\mathrm{F}^{-}$

6. $\mathrm{PPCO}$, excellent for $\mathrm{F}^{-}$

7. TPX, good for $\mathrm{F}^{-}$

8. PC, good for $\mathrm{F}^{-}$

9. PS, good for $\mathrm{F}^{-}$

In the hot water extraction procedure, PS could not be included due to the low softening temperature. In the hot water extraction procedure one would expect rapid elution, and stabilization of the material within 24-48 hours so that no further contamination occurs. 

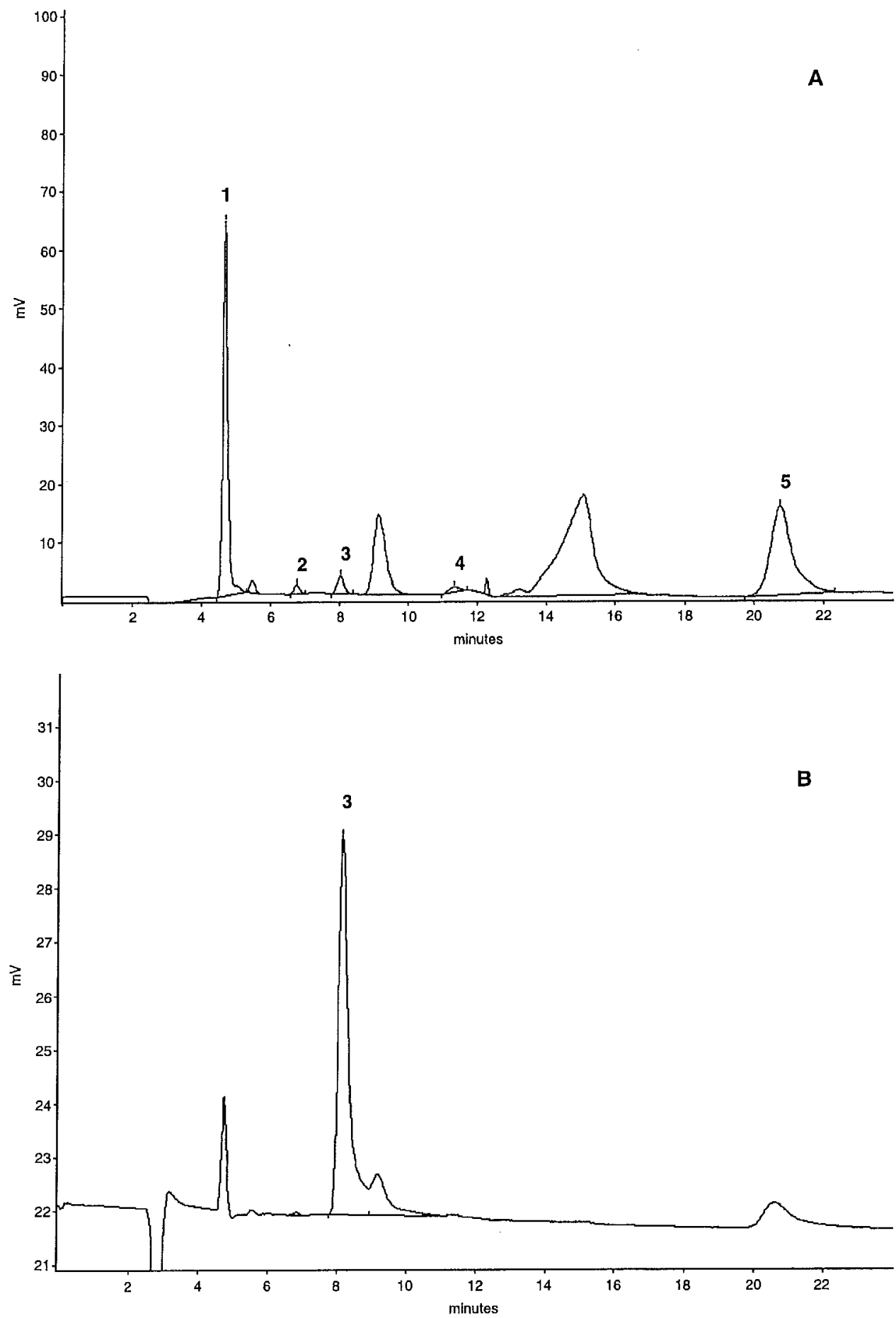

Fig. 2. Chromatogram of a FEP-eluted solution after 1 day of using cold water extraction. A) conductivity detector; B) electro-chemical detector. Legend: (1) Fluoride, (2) chloride, (3) nitrite, (4) nitrate, (5) sulphate

This assumption turned out to be incorrect upon experimental investigation. (Figs. 8-12). Fluoride showed high-level contamination up to $300 \mu \mathrm{g} \mathrm{L}^{-1}$ in FEP (Fig. 8), whereas the other materials showed contamination level below $40 \mu \mathrm{g} \mathrm{L}^{-1}$ after one week of elution. Figures 9-12 show that apart from nitrogen- 


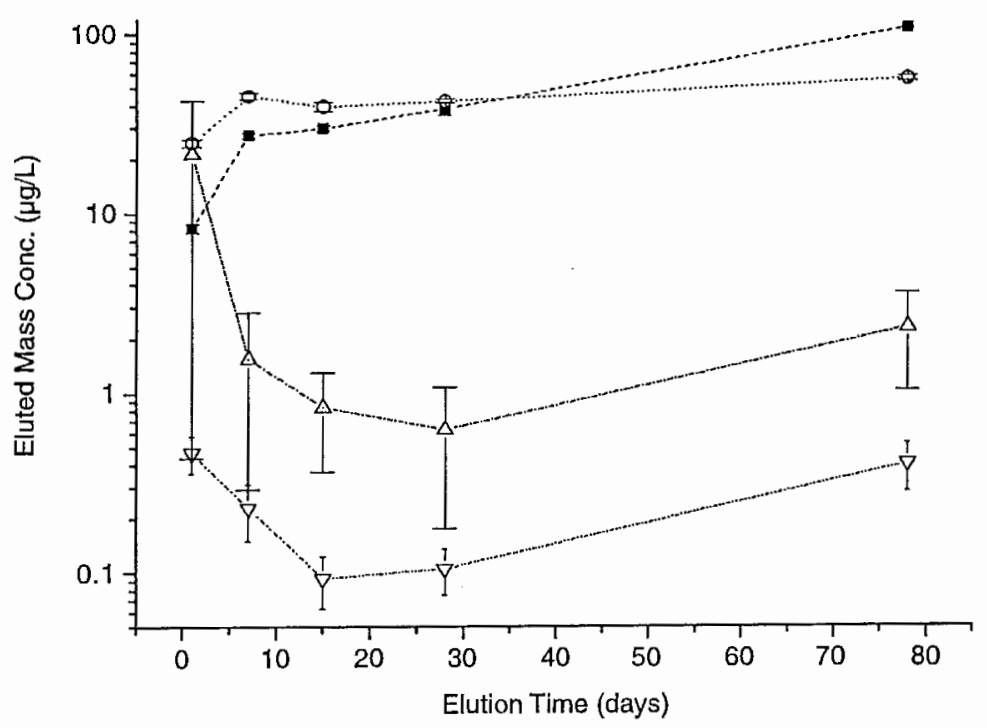

Fig. 3. Fluoride released into the eluate as a function of time under cold water extraction.

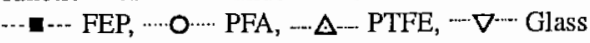
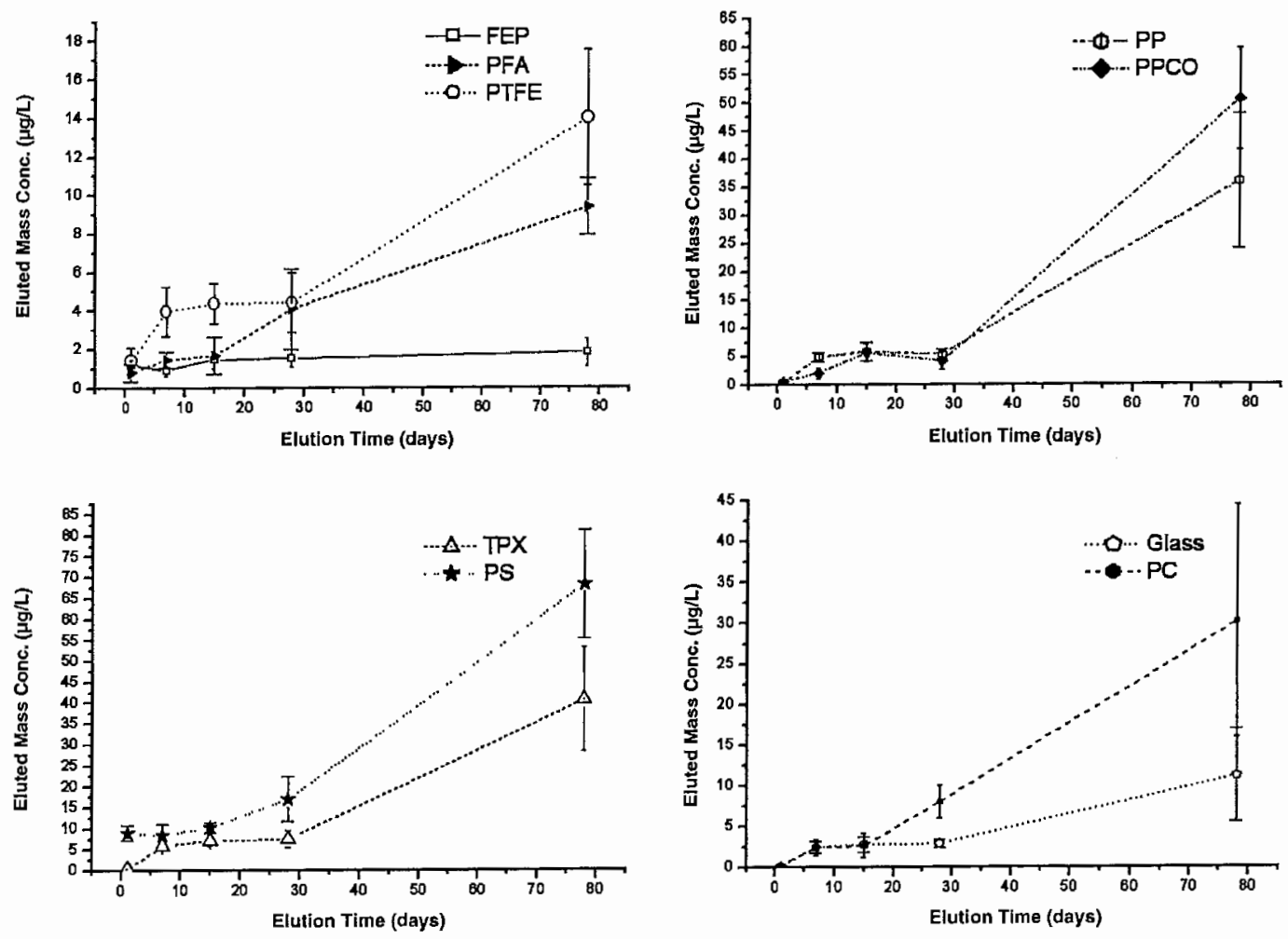

Fig. 4. Nitrite released into the eluate as a function of time under cold water extraction

based anions, all other anions increased steadily throughout the entire observation period. Nitrite was generally below $15 \mu \mathrm{gL}^{-1}$. Similarly, nitrate was mostly below $15 \mu \mathrm{g} \mathrm{L}^{-1}$ except in the case of FEP, PFA and TPX, where more variable values were found. Chloride was continuously eluted for the whole observation period, up to $35 \mu \mathrm{g} \mathrm{L}^{-1}$ in TPX. Sulphate showed a greater material-dependence, with most of the material producing contamination below $5 \mu \mathrm{g} \mathrm{I}^{-1}$, and TPX and glass yielding up to $75 \mu \mathrm{gL}^{-1}$. 

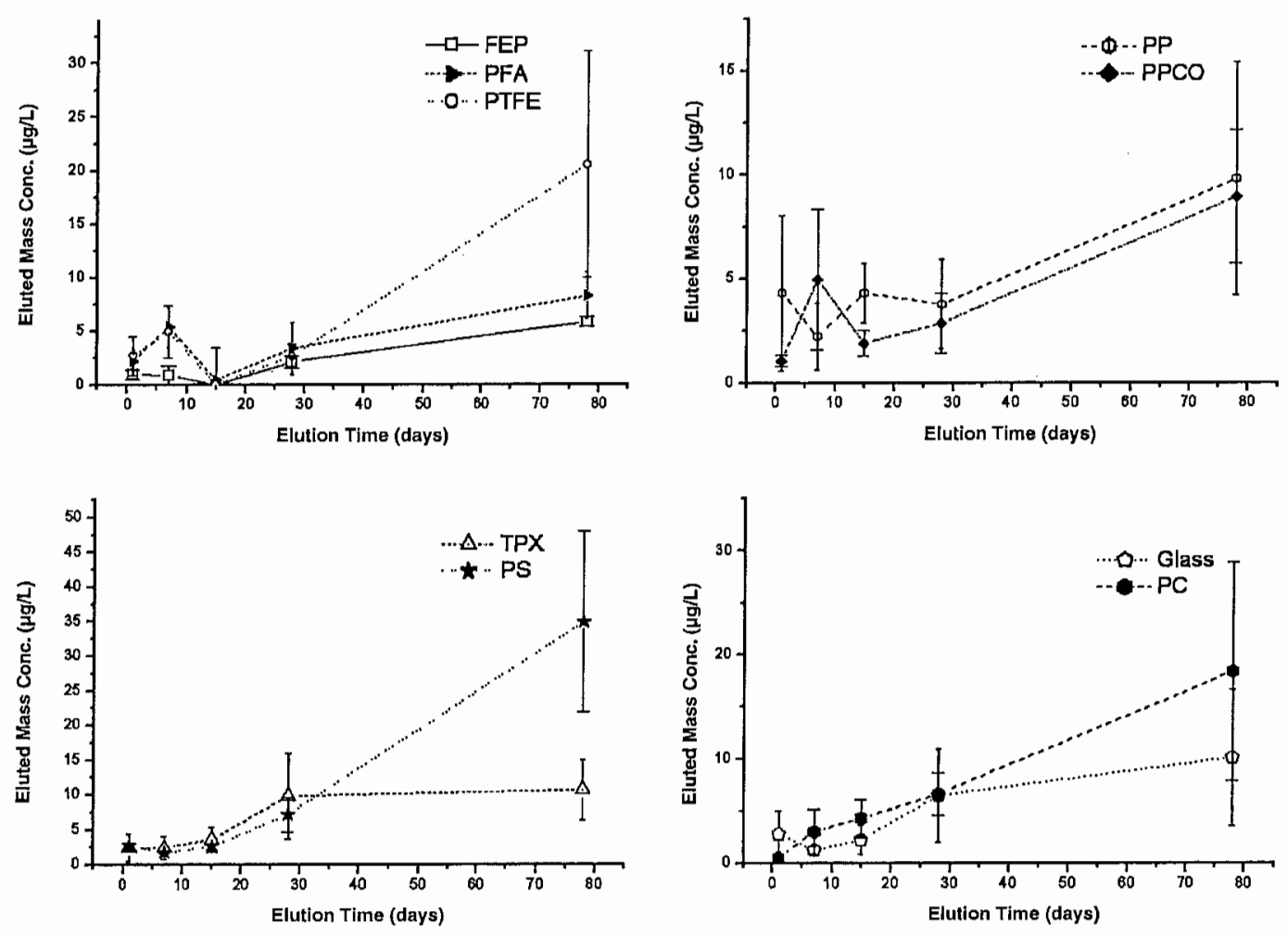

Fig. 5. Nitrate released into the eluate as a function of time under cold water extraction
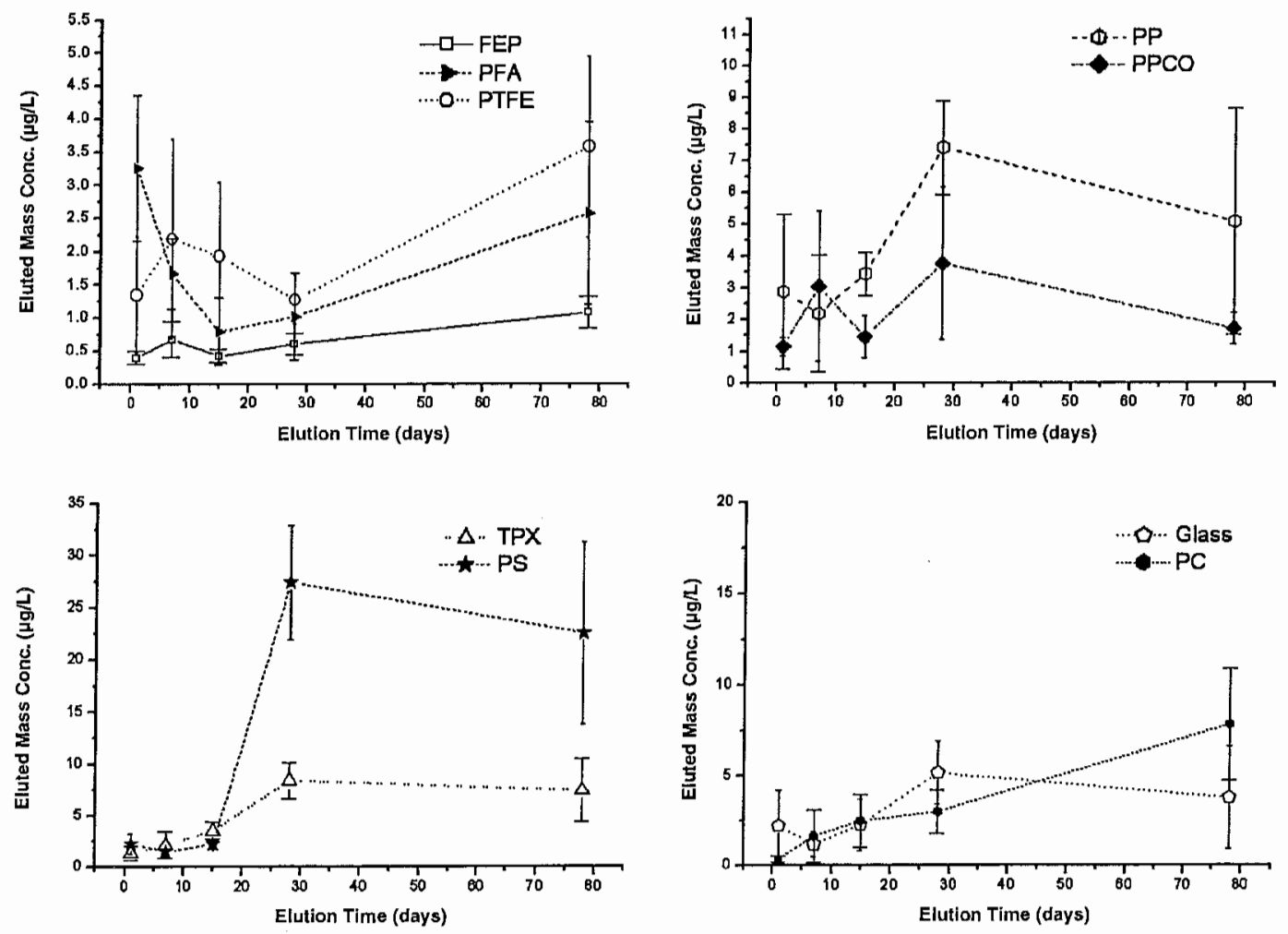

Fig. 6. Chloride released into the eluate as a function of time under cold water extraction 
R. Figi et al.
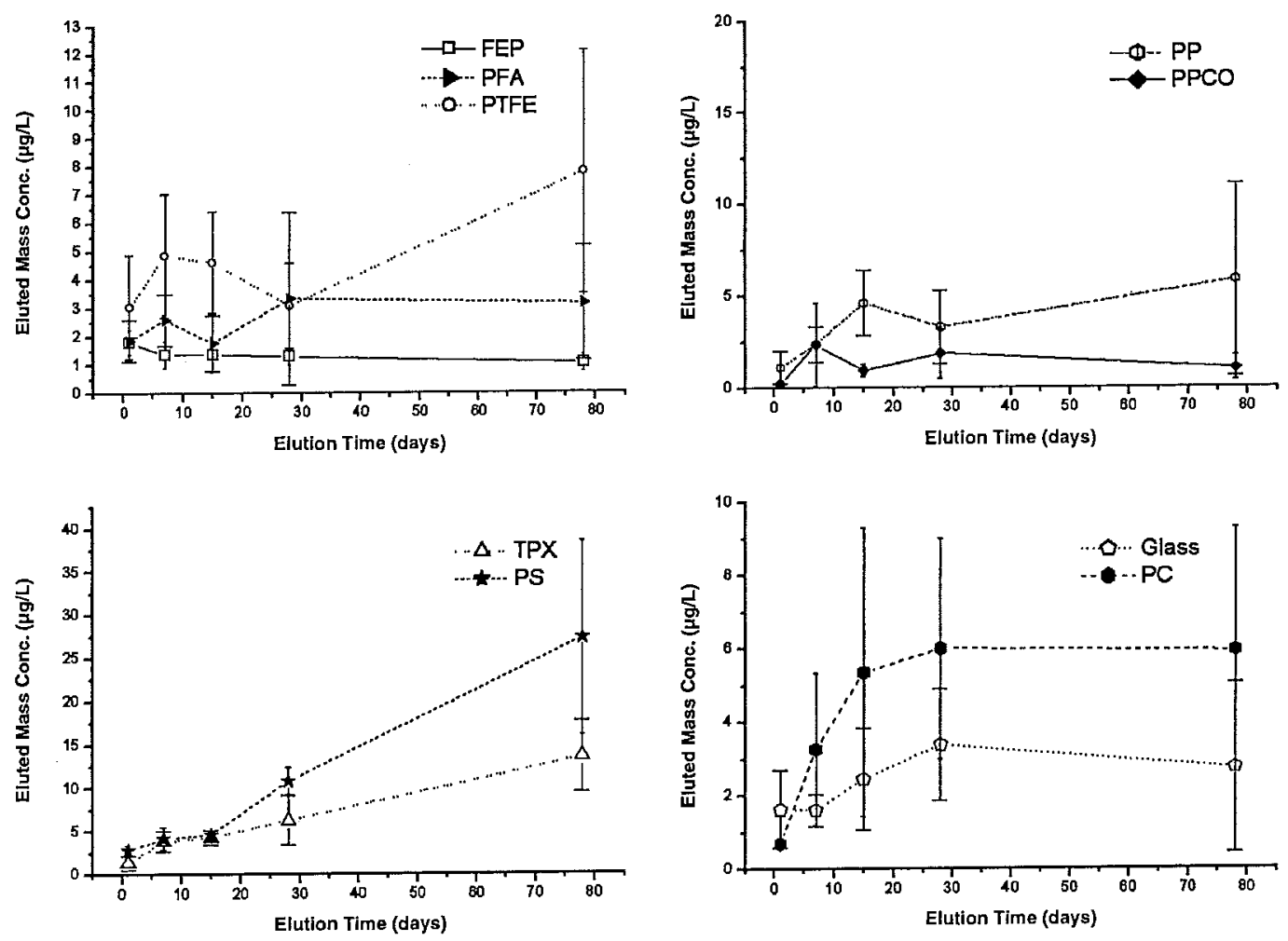

Fig. 7. Sulphate released into the eluate as a function of time under cold water extraction

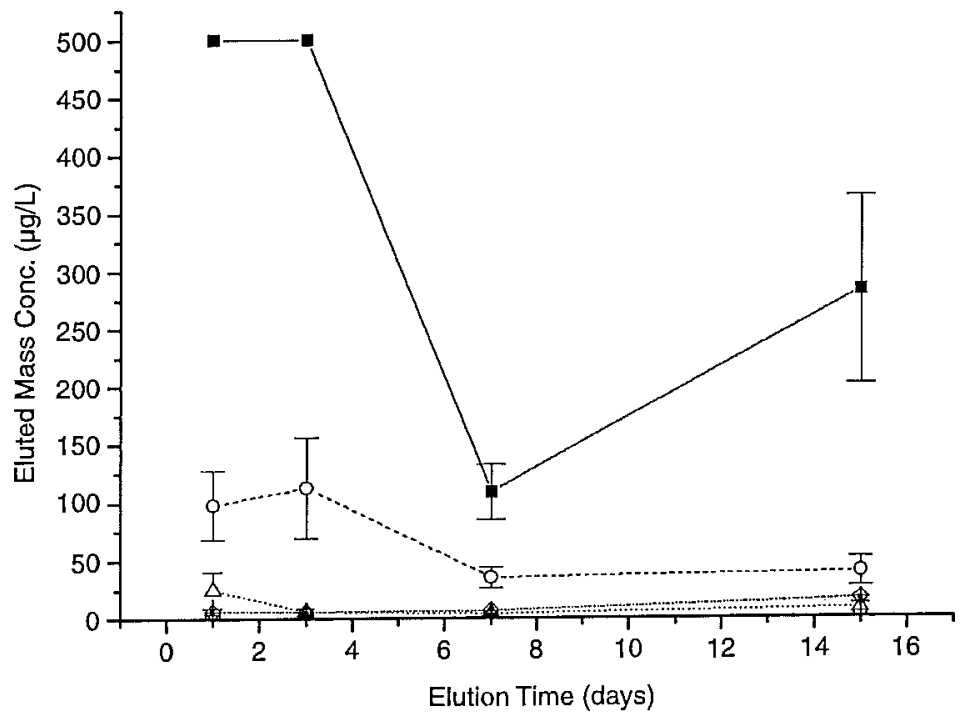

Fig. 8. Fluoride released into the eluate as a function of time under hot water extraction. -...-.. FEP,

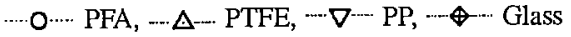

Material performance under hot water extraction could be ranked as follows:

1. FEP, all anions with the exception of $\mathrm{F}^{-}$

2. PTFE, good for $\mathrm{NO}_{3}{ }^{-}$

3. PFA, all anions with the exception of $\mathrm{F}^{-}, \mathrm{NO}_{3}{ }^{-}$
4. $\mathrm{PP}$, good for $\mathrm{F}^{--}$and $\mathrm{SO}_{4}{ }^{2-}$ only

5. Glass 950-1, all anions with the exception of $\mathrm{F}^{-}$, $\mathrm{NO}_{2}{ }^{-}, \mathrm{SO}_{4}{ }^{2-}$

6. PPCO, excellent for $\mathrm{F}^{-}$

7. $\mathrm{PC}$, good for $\mathrm{F}^{-}$only

8. TPX, good for $\mathrm{F}^{-}$and $\mathrm{NO}_{2}^{-}$ 

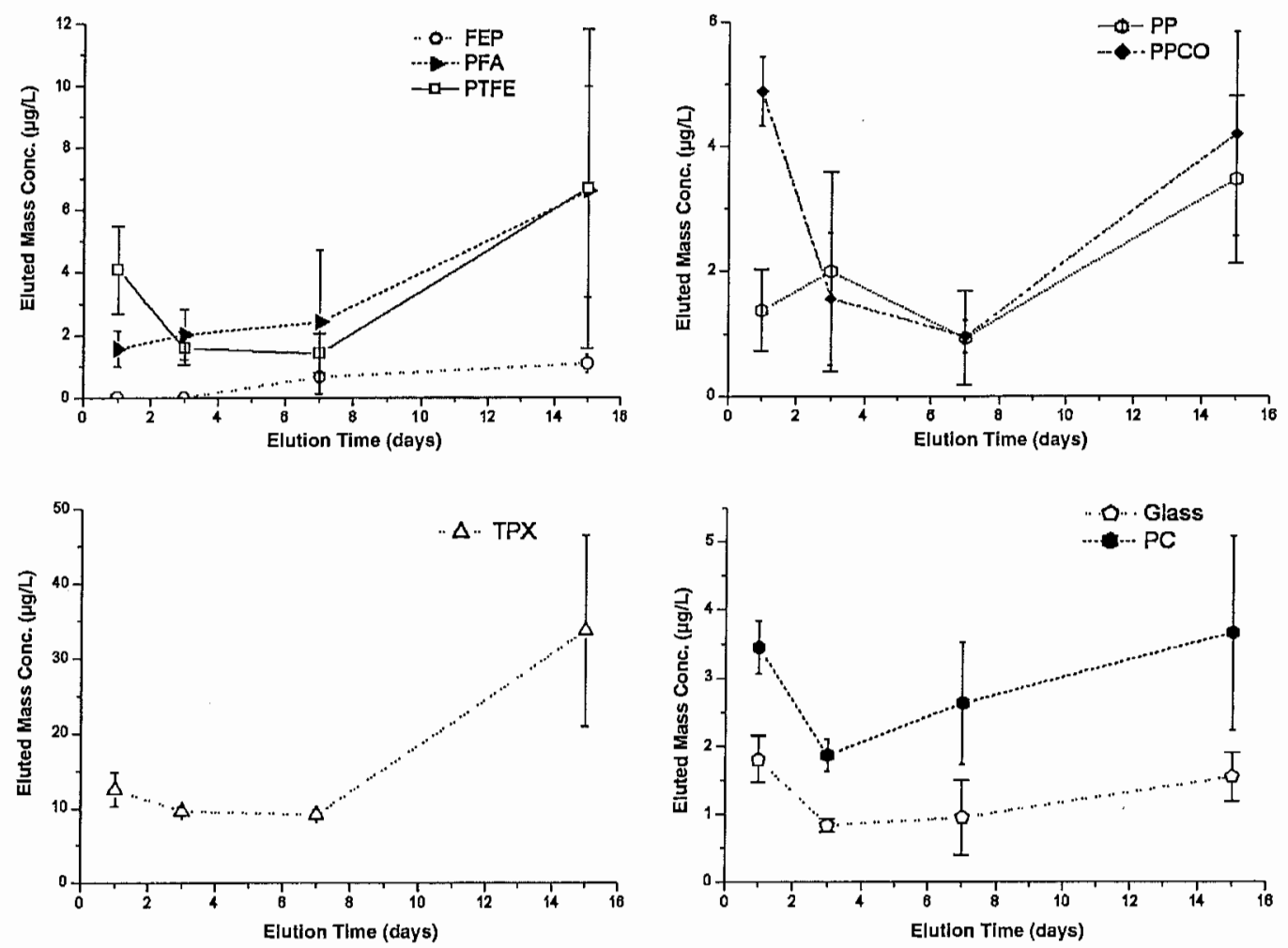

Fig. 9. Chloride released into the eluate as a function of time under hot water extraction
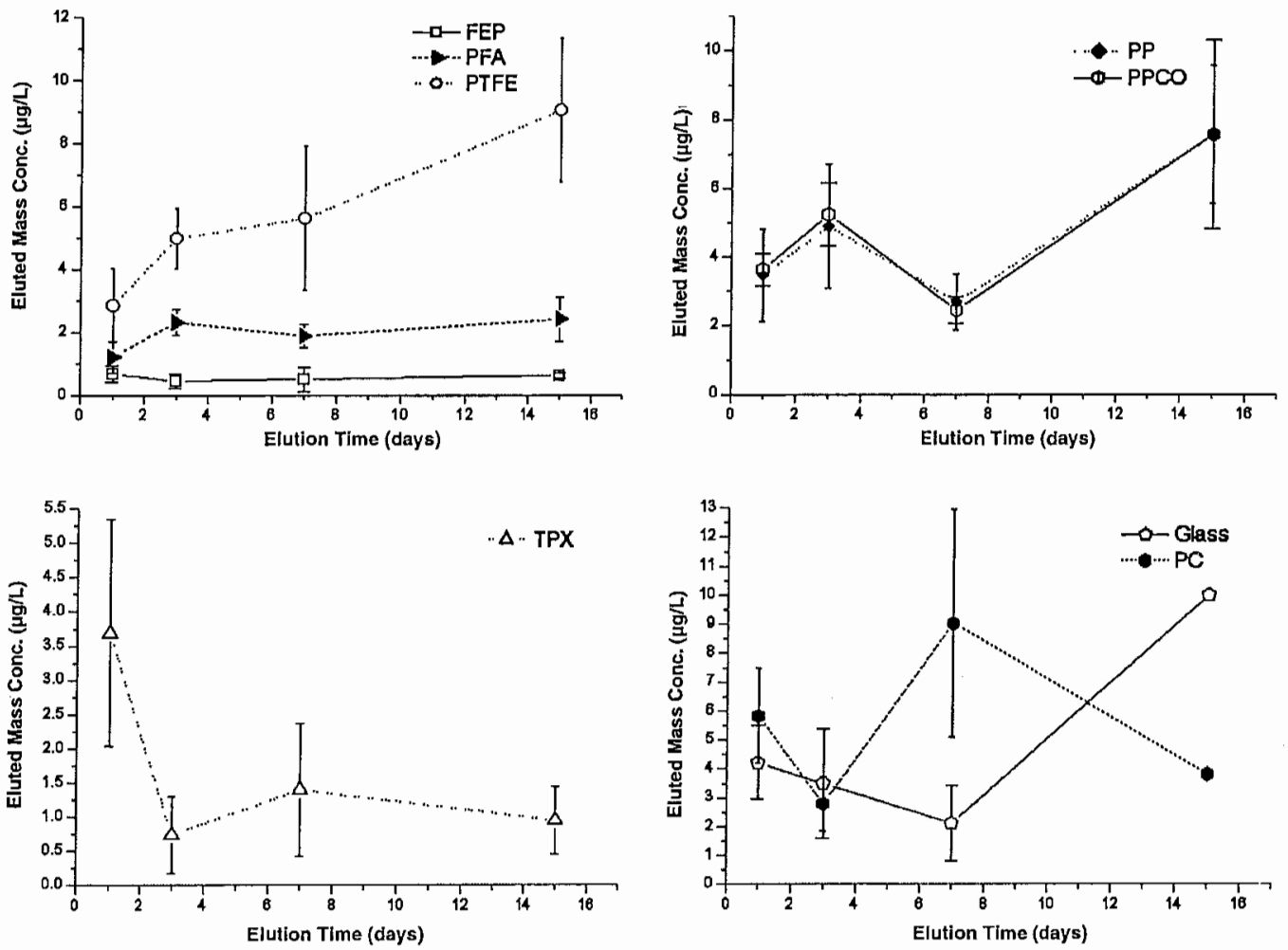

Fig. 10. Nitrite released into the eluate as a function of time under hot water extraction 

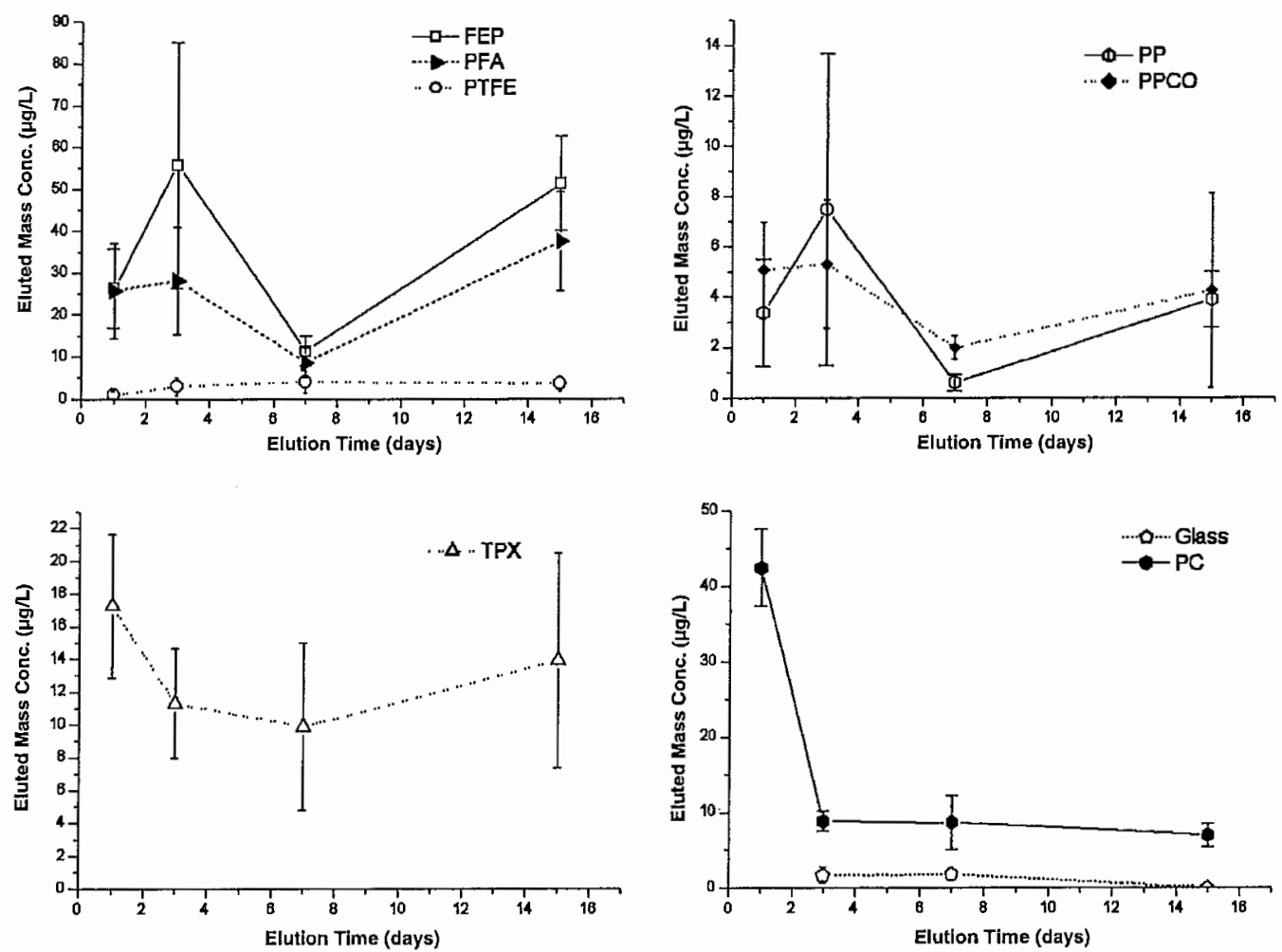

Fig. 11. Nitrate released into the eluate as a function of time under hot water extraction
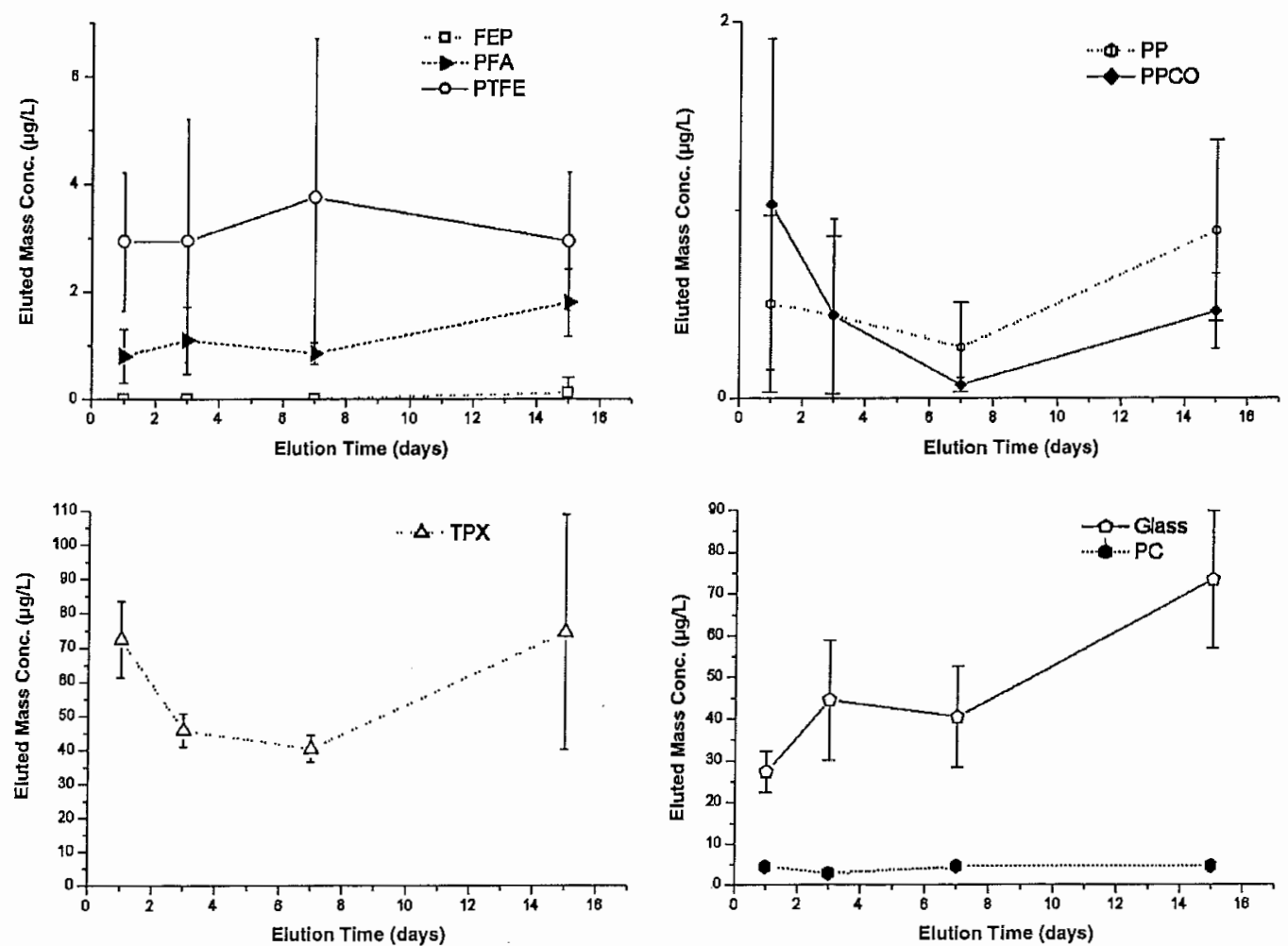

Fig. 12. Sulphate released into the eluate as a function of time under hot water extraction 
Hot water extraction generally provided less satisfactory performance than the above discussed cold water extraction, so that the latter ranking list should be considered subordinate to the one previously presented.

\section{Conclusion}

Semiconductor industries require lower and lower detection limits for anions which are hardly attainable by improvements in instrumental sensitivity. Therefore, more efforts must be made to improve the blank quality. The procedure developed here pursues this goal, presenting the suitability of different plastic vial materials for different anion ultratrace analyses with IC. A systematic investigation of the performance of several commercially available sample vial materials showed that the material should always be selected with respect to the analyte anion of interest. Nevertheless, there is no vial material perfectly suited for storage of any ultra-pure liquid sample or for analysis of any ultra-trace anion. The user is recommended to select the appropriate vial specifically for her/his application, considering the performance trends of individual materials shown here. Attention must be paid to the fact that contamination is a dynamic process. For instance, during auto-sampler operation the endmost vials in the measurement sequence will be characterized by higher contamination than those at the beginning due to the elution process. In fact, the time-dependence of the different materials demonstrated the importance of short storage periods. Samples should not be stored for more than few hours prior to measurement.

In cold water extraction, fluorinated materials showed the most satisfactory results for anion analysis, except for the analysis of $\mathrm{F}^{-}$itself. In this case, PP and PPCO showed the best performance. Hot water extraction as a sample preparation procedure for fast elimination of contaminants is not recommended, and cold water extraction in combination with five rinses is the preferred method. In fact, all materials showed marked time-dependence of anion contamination into the solution. The sample volume critically influences the level of contamination because less sample volume means less contaminant dilution. The SEMI F40 procedure implies the availability of low sample amount for the measurements, which limits the lowest attainable DL as a consequence of blank purity.

Recent advances in the available instrumentation (Metrohm inline calibration system) will further improve DL, because the calibration standards can be prepared online by the system, so that no storage is required. This way potential contamination induced by sample vials can be overcome.

Acknowledgements. U. Waldburger (Metrohm, Switzerland) is acknowledged for support in instrumentation and know-how $O$. Nagel and A. Wichser (EMPA) provided valuable help throughout the project. Thanks also to H. Vonmont (EMPA) for a critical review of this manuscript.

\section{References}

[1] The annual book of ASTM standards (2003) Standard practice for handling of ultra-pure water samples. D 4453-02, p 475

[2] Semi F40-0699 practice for preparing liquid chemical distribution components for chemical testing

[3] Semi F57-0301 provisional specification for polymer components used in ultrapure water and liquid chemical distribution systems

[4] Nguyen VD (1996) Fres J Anal Chem 354: 738

[5] Nguyen VD, Neumeister H, Subklew G (1999) Fres J Anal Chem 363: 783

[6] Vanatta LE (1996) J Ion Chromatogr A 739: 199

[7] International Organization for Standardization ISO 14644-2: 2000 Cleanrooms and associated controlled environments Part 2: specifications for testing and monitoring to prove continued compliance with ISO 14644-1 
